\title{
Huella del Carbono. Parte 2: La Visión de las Empresas, los Cuestionamientos y el Futuro
}

\author{
César Espíndola ${ }^{(1,2)}$ y José O. Valderrama ${ }^{(3,4)}$ \\ (1) Univ. de La Serena, Fac. de Ingeniería, Dpto. de Ing. Industrial, Casilla 554, La Serena-Chile \\ (2) Univ. de Lleida, Depto. Adm. Empresas y Gestión Económica de Rec. Naturales, Lleida-España \\ (3) Univ. de La Serena, Fac. de Ingeniería, Dpto. de Ing. Mecánica., Casilla 554, La Serena-Chile \\ (4) Centro de Información Tecnológica (CIT), Casilla 724, La Serena-Chile
}

Recibido Sep. 10, 2011; Aceptado Oct. 28, 2011; Versión Final recibida Dic. 02, 2011

\begin{abstract}
Resumen
En esta segunda parte de la serie, se discute sobre la visión y posición que tienen las empresas frente al concepto de Huella del Carbono $(\mathrm{HdC})$ y de los métodos para cuantificarla. Se analiza los criterios de clasificación de emisiones en las principales metodologías de cálculo de la HdC y se discute sobre los principales cuestionamientos a la HdC, sobre los temas a resolver y sus alternativas de solución. Se presenta una base comparativa para los cuatro principales métodos de determinación de $\mathrm{HdC}$ actualmente vigentes en el mercado y se propone un método denominado Abaco para la contabilización de emisiones de $\mathrm{CO}_{2}$ equivalente, que permite identificar las características comunes de estos métodos. Todo esto puede servir de base en la toma de decisiones gerenciales para adoptar una determinada metodología. Se concluye que de mantenerse la falta de claridad y comparabilidad en la determinación de emisiones podría provocar la pérdida de una gran oportunidad para lanzar una nueva economía medioambientalmente sostenible.
\end{abstract}

Palabras clave: huella del carbono, $\mathrm{HdC}$, visión de las empresas, $\mathrm{CO}_{2}$ equivalente.

\section{Carbon Footprint: Part 2: Enterprises Viewpoint, Doubts and the Future}

\begin{abstract}
In this second part of the series, the viewpoint of the enterprises and their position with respect to the implementation of carbon footprint (CFP) quantification. The criteria for classification of emissions and the main methodologies for calculating the CFP are discussed and analyzed. The main questions and doubts about the CFP, on the subjects to be discussed and the alternatives of solution. A comparison of the main four methods currently used for determining the CFP and a method named Abacus for quantifying emissions of equivalent $\mathrm{CO}_{2}$ that allows identifying the common characteristics to all of them. All this can serve as basis for decision-making to adopt a given methodology. It is concluded that if the present situation of confusion of the different approaches to quantify the CFP continues, the companies and governments are loosing a great opportunity to arrive to a new environmentally sustainable economy.
\end{abstract}

Keywords: carbon footprint, CFP, enterprise viewpoint, equivalent $\mathrm{CO}_{2}$. 


\section{INTRODUCCIÓN}

Como se describe en la Parte 1 de esta serie, la Huella del Carbono (HdC), se perfila como un indicador capaz de sintetizar los impactos provocados por las actividades del hombre en el entorno, medido en términos de emisiones de gases efecto invernadero (GEI) y se presenta como una poderosa herramienta de gestión y un estímulo para adoptar una estrategia proactiva en el logro de la sustentabilidad de las organizaciones. (Wiedmann y Minx, 2008, Boiral, 2006; Wittneben y Kiyar, 2009). En particular, el uso de la HdC ha encontrado un importante campo de contribución como es la eficiencia energética y su impacto en los costos operacionales de la empresa, situación que mejora el margen de beneficios de la empresa contribuyendo no sólo a la sustentabilidad ambiental, sino también, a la rentabilidad económica de la misma- El éxito en la contribución al cambio climático que pueda ofrecer la HdC para poner en práctica cadenas de producción sostenibles, pasa por el reconocimiento de las responsabilidades de productores y también de consumidores. Esto porque si el consumidor sigue exigiendo un producto o servicio a un precio que produce pobres resultados ambientales e injustos efectos sociales y económicos, se requerirá mayor esfuerzo de las empresas para convencer y mejorar las decisiones de compra del consumidor. Sin embargo, incluso una empresa que comience exitosamente como "la más verde de las empresas" estará forzada a afrontar las realidades del mercado (Foran et al., 2004).

El desarrollo de métodos para calcular las emisiones de carbono ha sido relativamente rápido y se han ido adaptando a las diversas políticas gubernamentales y las agendas corporativas (Plassmann et al., 2010). Sin embargo, tan diversos intereses han impedido la adopción de una forma general para su cuantificación. Los principales intentos han sido patrocinados por gobiernos que buscan facilitar la definición de estándares nacionales que apuntan al cumplimiento de los compromisos de emisión adquiridos. Por cierto, la HdC comienza a perfilarse como un útil mecanismo de protección para la competitividad de las producciones nacionales y un medio simple a la entrega de información a los consumidores. Esto está produciendo cambios en los precios relativos y en los costos de emitir carbono. Sin embargo, más importante que esto son las modificaciones en los patrones de producción y consumo que se está produciendo y que pueden dar origen a nuevas oportunidades de negocios o pérdidas de competitividad para las empresas (De La Torre et al., 2009). Una forma de entregar esta información es el correcto etiquetado de los productos indicando en la etiqueta la magnitud de la $\mathrm{HdC}$ de dichos productos (Plasmann et al., 2010, Röös et al., 2010).

La introducción de la $\mathrm{HdC}$ como un indicador en la gestión de cadenas de suministro impone un importante desafío a los países en vías de desarrollo dado que gran parte de los productos que se consumen en Europa, Norteamérica et al. países industrializados, se producen en países en desarrollo. Esta situación, unida a la falta de conocimientos científicos sobre las emisiones de GEI de los países en desarrollo, implica para los países desarrollados un riesgo de que el etiquetado y que los instrumentos utilizados en la contabilidad de carbono no representen adecuadamente los sistemas de producción. Por otra parte, desde la perspectiva de los países en desarrollo el contar con una magnitud de $\mathrm{HdC}$ se transforma en factor de competitividad importante, dado que es un indicador que de estar ausente podría perjudicar la generación de beneficios económicos y/o una reducción de las oportunidades de exportación, al enfrentarse a restricciones al comercio. (Mcgregor y Vorley, 2006; Brenton et al., 2009; Kasterine y Vanzetti, 2010; Schneider y Samaniego, 2009).

No sólo la ausencia de declaración de emisiones constituye un riesgo, sino también la magnitud de este indicador, especialmente cuando ésta constituye una declaración de superioridad 0 equivalencia de un producto versus otro producto de la competencia. (SETAC, 2008). Diferentes metodologías, normas públicas como iniciativas privadas voluntarias para calcular la $\mathrm{HdC}$ de los productos, se encuentran operativas o en desarrollo, especialmente en países tales como el Reino Unido, Francia Alemania, Japón, Suiza, Suecia, Nueva Zelanda, EE.UU., Corea y Tailandia. (Schneider y Samaniego, 2009). Aunque ya se están aplicando varios esquemas, el estándar PAS 2050 (BSI, 2008) es el único completamente terminado y operativo para el cálculo de HdC para productos. El British Standard Institute (BSI) presentó en Londres en el mes de mayo de 2010 la norma PAS 2060, que se aplicará a organizaciones y productos que quieran convertir sus 
emisiones de $\mathrm{CO}_{2}$ en neutras. Esta certificación tiene como objetivo suplir un vacío que estaba generando confusión en el público, ya que cada vez hay más productos en el mercado que afirman ser neutros en emisiones y no había ninguna norma en esta materia (BSI, 2010). Algunos sectores interesados consideran que incluso si una norma internacional logra ser desarrollada como el caso de la ISO 14067 Carbon Footprint, la demanda de requisitos más específicos podría limitar la adopción de la HdC. Esto generaría la adopción de normas de nivel nacional o de ciertos sectores comerciales, en especial si la norma ISO no define claramente las reglas de clasificación de productos, a fin de comparar bienes o servicios con alcances similares en los ciclos de vida y que tengan etapas similares de producción, entre otros factores (Röös et al., 2010).

En el caso de las economías abiertas, la $\mathrm{HdC}$ no sólo trae consigo el establecimiento de parámetros que permiten discriminar a favor o en contra de la demanda de los productos, sino que impone importantes desafíos de competitividad al sector exportador, sin importar el rubro del que se trate. Las emisiones de GEl asociadas al transporte de productos hacia los mercados de destino, en teoría, constituirían uno de los principales parámetros diferenciadores entre bienes de consumo similares. De consolidarse esta tendencia mundial en el comercio internacional, se establecería una desventajosa y negativa percepción de los productos provenientes de países más lejanos a los distintos puntos de consumo final, dada su alta intensidad de carbono esperada producto de las emisiones asociadas al transporte (Schneider y Samaniego, 2009). Sin embargo las emisiones del proceso productivo en muchos casos pueden superar a las del transporte. En América Latina, sin embargo, el tema apenas empieza a reconocerse y pocos han comenzado a asumir iniciativas específicas para cuantificar la HdC de los productos de exportación como estrategia preventiva, reconociéndose la ausencia de definiciones sobre el marco metodológico a utilizar en el cálculo, la determinación de factores de conversión locales, la generación de datos propios de emisión, la identificación de fases críticas de los procesos y cadenas de suministro de bienes y servicios. Las iniciativas existentes responden a las nuevas exigencias de los mercados compradores o de empresas transnacionales (Schneider y Samaniego, 2009).

Entre los países que destacan por sus esfuerzos en la determinación de la HdC y su futura adopción se cuentan Alemania, Estados Unidos, Reino Unido, Japón y Francia quienes han logrado importantes avances. De este grupo de países Francia es probablemente el país que ofrece menores horizontes de tiempo para que sus iniciativas deriven en acciones mandatorias y legales que establezcan obligatoriedad respecto de la información de la $\mathrm{HdC}$ a ser entregada a la ciudadanía. Esto agregará sin dudas un nuevo factor a considerar en el proceso de decisión de compra y consumo de bienes y servicios, que por años ha estado dominada por el precio y su relación con la utilidad (Ademe, 2010). La HdC comienza a ganar importante terreno en la construcción del valor de productos y servicios.

\section{LA VISIÓN DE LAS EMPRESAS}

De mantenerse esta falta de claridad y comparabilidad en la determinación de emisiones podría provocar a la larga el fracaso de un eco-etiquetado global y la pérdida de una gran oportunidad para lanzar una nueva economía sostenible (Dómenech et al., 2010). La literatura recalca la importancia de que los tomadores de decisiones, entiendan tanto las diferencias en los enfoques adoptados para la cuantificación de la $\mathrm{HdC}$ así como de las complejidades metodológicas inherentes a cada método, y como éstos pueden influir en los resultados del valor obtenido. En el ámbito del comercio internacional hay dos enfoques que han cobrado importancia: i) el enfoque de producto basado fundamentalmente en el análisis el ciclo de vida de dicho producto; y ii) el enfoque corporativo (Weidmann, T et al. 2007).

Ambos enfoques metodológicos podrían generar importante repercusión a corto plazo en los países de América Latina, fundamentalmente por el carácter eminentemente exportador de recursos naturales y productos de bajo valor agregado, como son los productos agrícolas, forestales, acuícolas y mineros. Por otra parte, cuando una empresa se enfrenta a más de un mercado en los cuales rigen diversas metodologías de cálculo, la incertidumbre se incrementa y surge el cuestionamiento de dicho indicador y su veracidad respecto de la relación con el ambiente (Weidmann, 2009; BOLWIG Y GIBBON, 2009).. En este sentido, parece relevante 
abordar desde Latinoamérica procesos de investigación destinados a resolver interrogantes frente a comparación de enfoques metodológicos de cálculo, en especial, asociados los métodos más relevantes analizados en la Parte 1 de esta serie (PAS 2050, GHG, Bilan Carbone, MC3), además del futuro estándar ISO14067.

\section{Principales cuestionamientos a la HdC}

El reflejo del amplio espacio para el desarrollo de nuevas investigaciones en ésta área está en los numerosos cuestionamientos y criterios a resolver que se detectan en la literatura. Entre estas se pueden mencionar: el ajuste de fronteras de sistema comparables, las exigencias en cuanto a la calidad de datos, la cobertura regional y tecnológica, reglas sobre cuando usar datos genéricos o específicos de un proceso como parte de la cadena de suministro del producto, factores de consumo de electricidad para el cálculo de la HdC y factores para el transporte, así como criterios para revisión crítica y contrato con los grupos de interés. Un resumen de estos criterios y sus cuestionamientos se presenta en la Tabla 1. La solución de este importante número de cuestionamiento hoy está cifrada en la aprobación internacional del estándar ISO 14067 y sus normas ISO 14067 Huella de Carbono de Productos Cuantificación y Comunicación, refundidos en un solo estándar que actualmente se encuentra en discusión.

Tabla 1: Principales criterios y cuestionamientos a la HdC (Setac, 2008; Plassmann et al., 2010; Sinden, 2009)

\begin{tabular}{|c|c|}
\hline Criterios a resolver & Alternativas de solución \\
\hline $\begin{array}{l}\text { 1.-Alcance de } \\
\text { emisiones }\end{array}$ & $\begin{array}{l}\text { - Considerar todos los GEl especificados por el IPCC en el año } 2007 \\
\text { - Considerar sólo los seis GEl incluidos en el Protocolo de Kioto }\end{array}$ \\
\hline $\begin{array}{l}\text { 2.-Etapas de ciclo de } \\
\text { vida }\end{array}$ & $\begin{array}{l}\text { - Considerar la etapa de utilización del producto en el análisis del ciclo de vida } \\
\text { - Resolver la polémica entre las perspectivas B2B (Negocio a Negocio) o B2C } \\
\text { (Negocio a Consumidor) } \\
\text { - Cómo hacer significativo el cálculo de HdC frente a estas perspectivas (B2B y } \\
\text { B2C) }\end{array}$ \\
\hline $\begin{array}{l}\text { 3.-Fronteras de } \\
\text { sistema }\end{array}$ & $\begin{array}{l}\text { - Especificar criterios para los límites del cálculo operacional, organizacional } \\
\text { - Considerar solamente emisiones directas, indirectas o subsidiarias } \\
\text { - Cómo incluir el transporte de los empleados } \\
\text { - Definir las fronteras de tiempo (anuales o asociadas al tiempo involucrado en el } \\
\text { ciclo de vida del producto). }\end{array}$ \\
\hline 4.-Compensación & $\begin{array}{l}\text { - Definir la inclusión o no, de la compensación en el cálculo de la HdC } \\
\text { - Definir la compensación por el uso de energía renovable }\end{array}$ \\
\hline 5.- Datos & $\begin{array}{l}\text { - Definir si los datos sólo deben estar asociados a procesos técnicos o deben existir } \\
\text { alguna conexión a los flujos de dinero } \\
\text { - Establecer las fuentes de datos (primarias o secundarias) } \\
\text { - Definir la calidad de los datos y la imposición de exigencias posibles }\end{array}$ \\
\hline 6.- Asignación & $\begin{array}{l}\text { - Establecer si el cálculo debe ser asignado por unidad temporal o monetaria } \\
\text { asignada al consumo u unidad de producto }\end{array}$ \\
\hline 7.-Final-de-vida & - Definir los criterios de final-de-vida y si se considera el reciclado \\
\hline $\begin{array}{l}\text { 8.-Almacenaje de } \\
\text { carbón }\end{array}$ & $\begin{array}{l}\text { - Definir cómo tratar el almacenaje de carbón (almacenaje geológico de carbono) } \\
\text { - Establecer como se consideran los procesos de captura de carbón }\end{array}$ \\
\hline $\begin{array}{l}\text { 9.- Cambio de uso de } \\
\text { suelo }\end{array}$ & $\begin{array}{l}\text { - Establecer si deben ser incluidas o no las emisiones por cambio de uso de suelo } \\
\text { - Definir si se consideran los sumideros de carbón del suelo }\end{array}$ \\
\hline $\begin{array}{l}\text { 10.-Generación de } \\
\text { datos comparables }\end{array}$ & $\begin{array}{l}\text { - Establecer criterios para que la HdC de los productos sean comparables } \\
\text { - Definir las unidades de medida de la HdC para los productos }\end{array}$ \\
\hline 11.-Bienes de capital & $\begin{array}{l}\text { - Definir que bienes de capital incluir } \\
\text { - Establecer cómo tratar los bienes de capital }\end{array}$ \\
\hline $\begin{array}{l}\text { 12.-Electricidad } \\
\text { renovable }\end{array}$ & $\begin{array}{l}\text { - Decidir si la electricidad renovable debe ser incluida o no } \\
\text { - Establecer si la matriz energética debe ser incluida por empresa o por sistema de } \\
\text { suministro }\end{array}$ \\
\hline
\end{tabular}




\begin{tabular}{|c|c|c|c|}
\hline \begin{tabular}{|r|r} 
& \\
$\frac{0}{0}$ \\
$\frac{\pi}{\omega}$ \\
0 \\
0 \\
0 \\
0 \\
0 \\
0 \\
0 \\
0 \\
\end{tabular} & 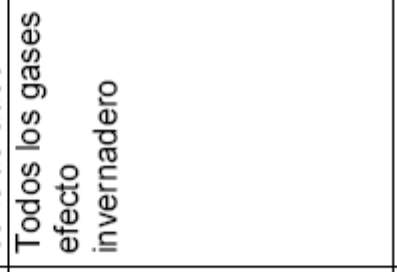 & 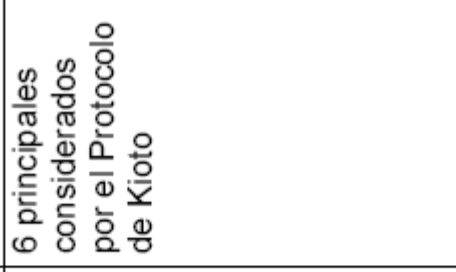 & 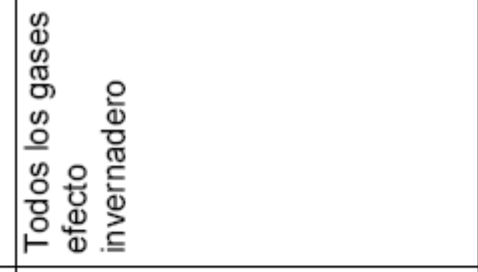 \\
\hline 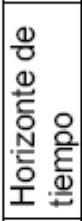 & 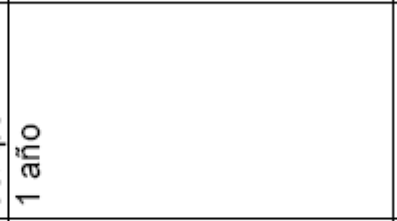 & 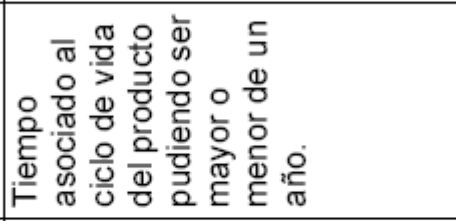 & 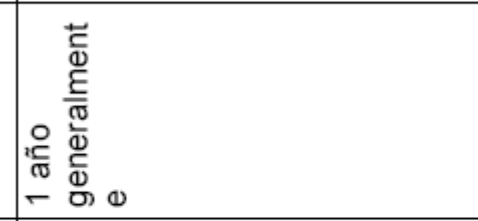 \\
\hline 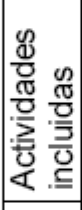 & 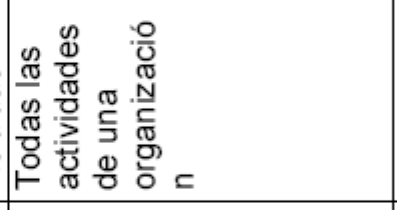 & 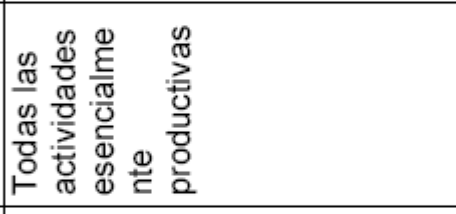 & 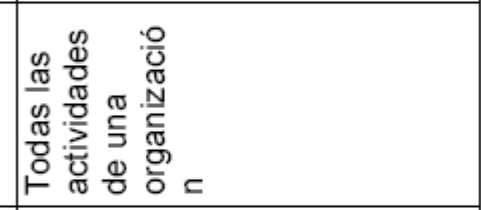 \\
\hline 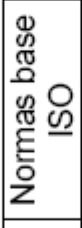 & 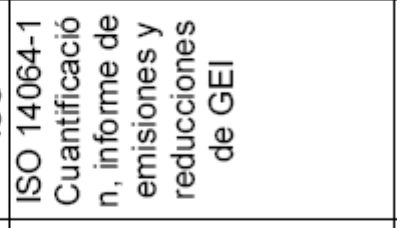 & 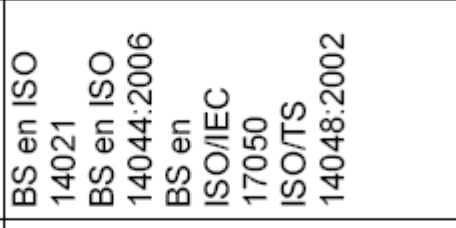 & 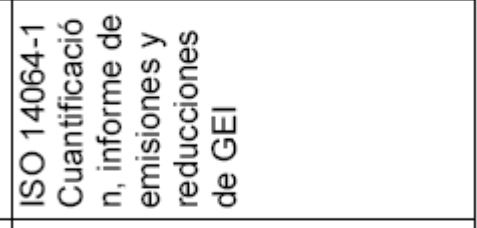 \\
\hline 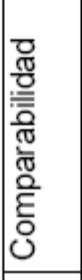 & 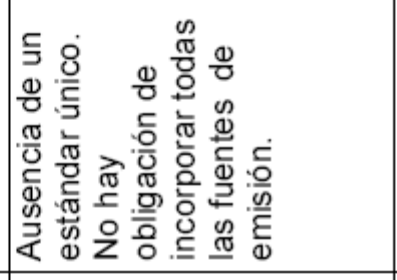 & 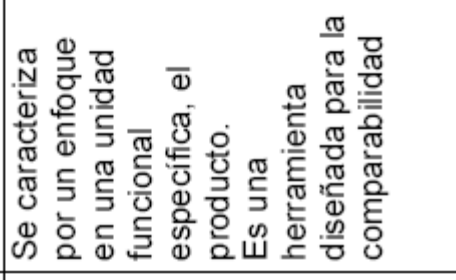 & 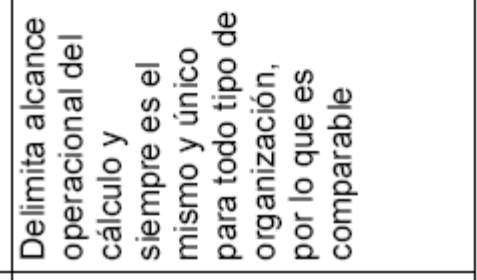 \\
\hline 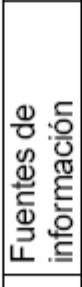 & 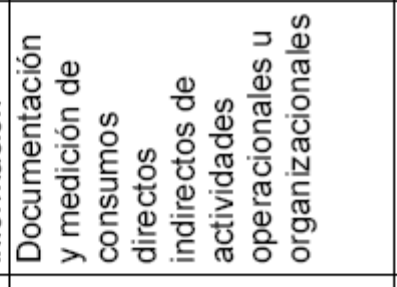 & 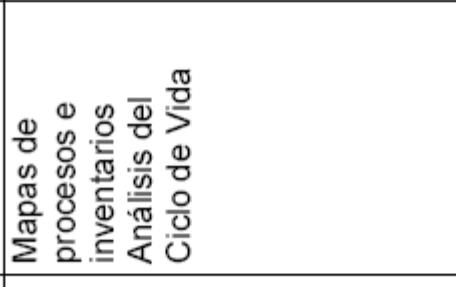 & 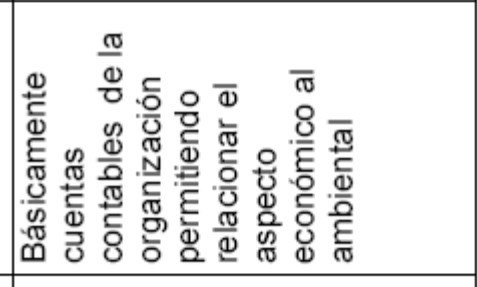 \\
\hline 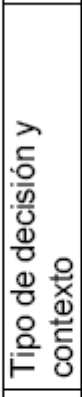 & 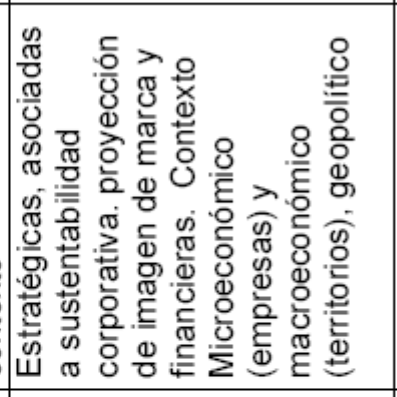 & 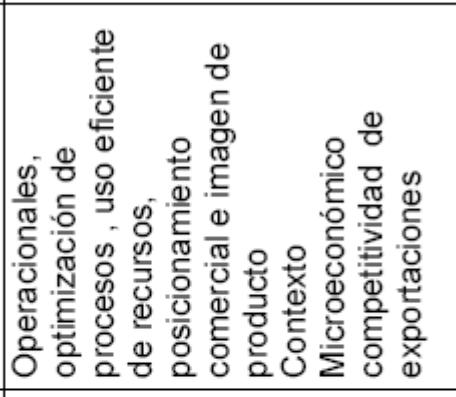 & 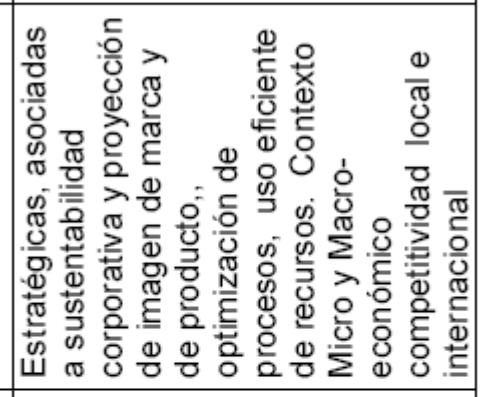 \\
\hline 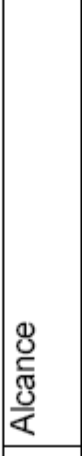 & 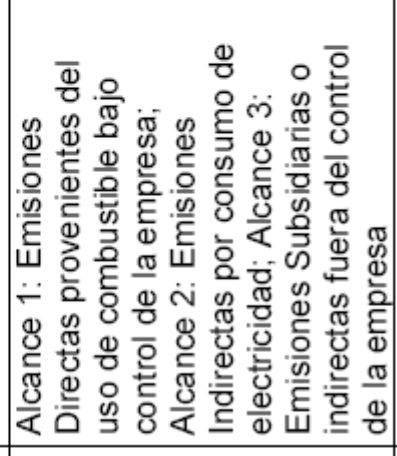 & 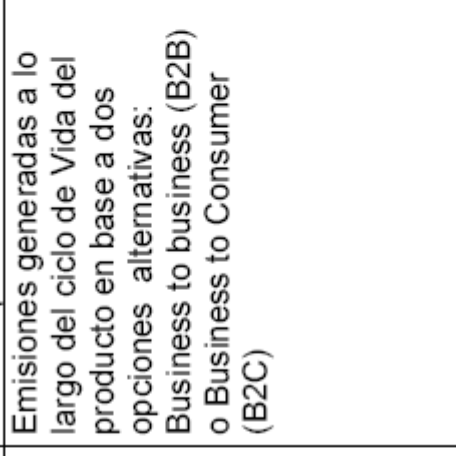 & 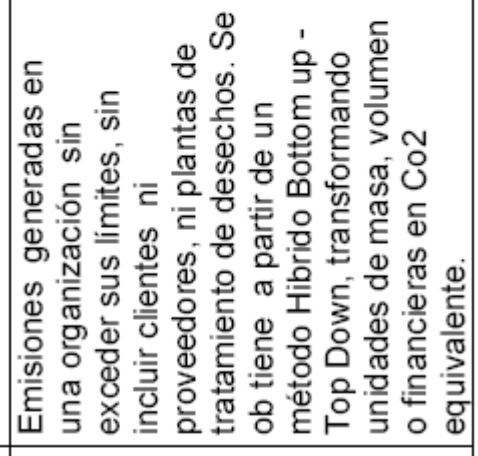 \\
\hline 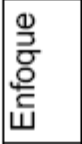 & 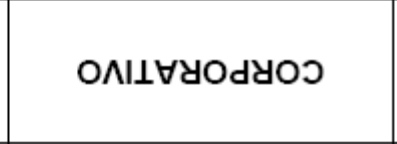 & ০เכnaoyd $\exists$ & 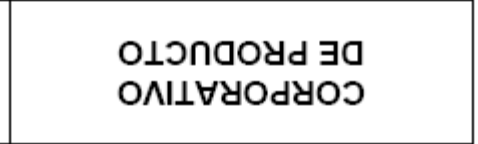 \\
\hline
\end{tabular}




\begin{tabular}{|c|c|c|c|c|c|}
\hline 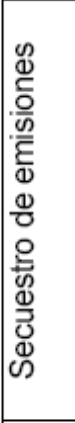 & $\begin{array}{l}\frac{\pi}{0} \\
\frac{0}{0} \\
\frac{0}{0}\end{array}$ & 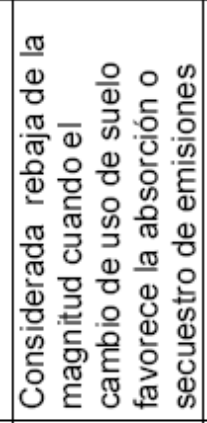 & 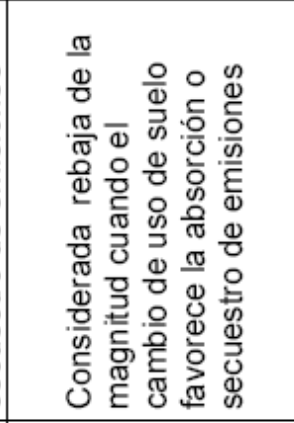 & 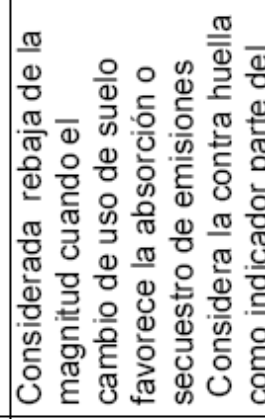 & 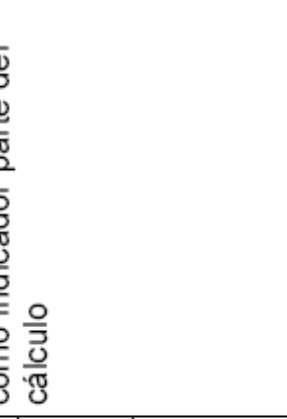 \\
\hline 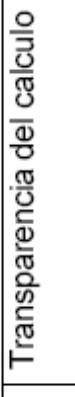 & 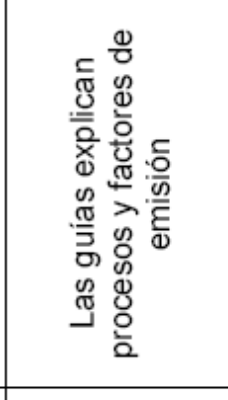 & 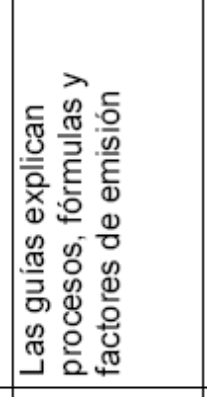 & 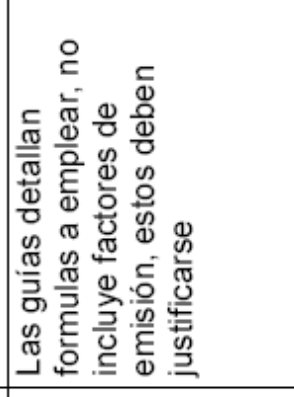 & 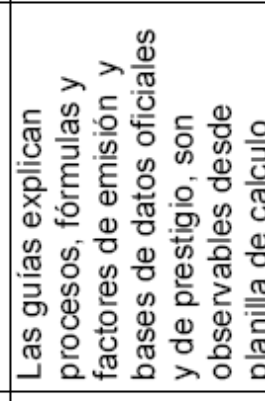 & 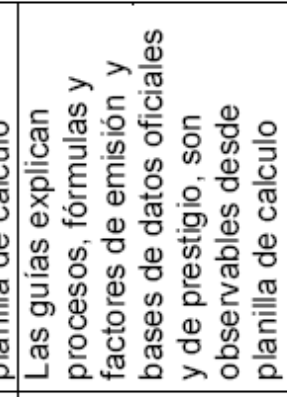 \\
\hline 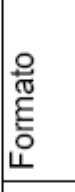 & 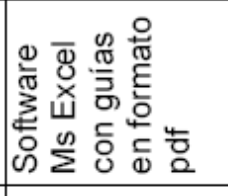 & 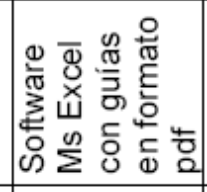 & 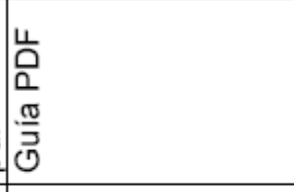 & 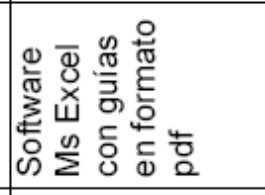 & 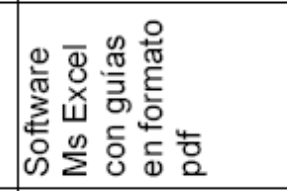 \\
\hline  & & 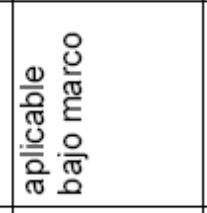 & 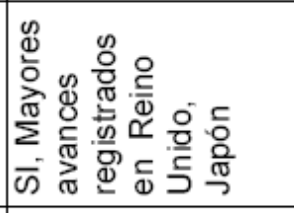 & 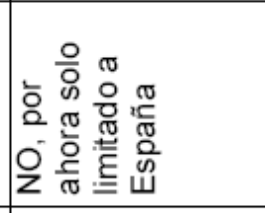 & 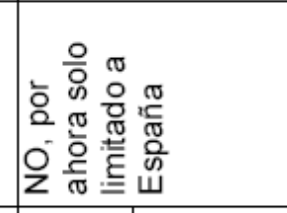 \\
\hline 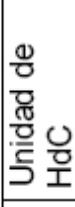 & 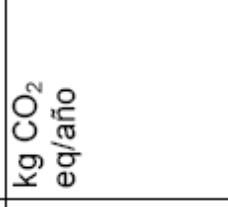 & 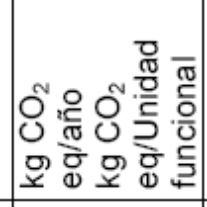 & 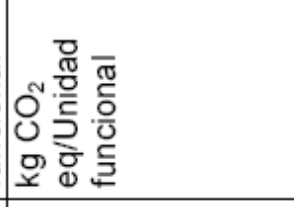 & 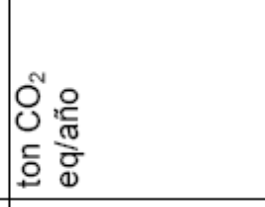 & 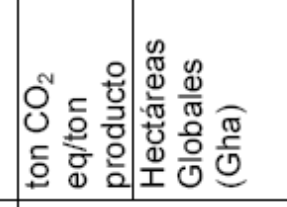 \\
\hline \begin{tabular}{|l}
$\frac{\pi}{\widetilde{d}}$ \\
出 \\
\end{tabular} & 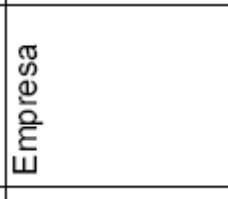 & 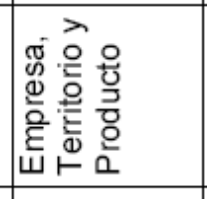 & $\begin{array}{l}\text { 융 } \\
\frac{2}{0} \\
\text { 은 } \\
\end{array}$ & 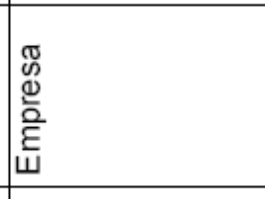 & 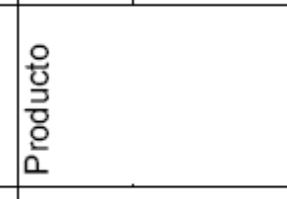 \\
\hline 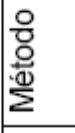 & 옹 & 옹 & 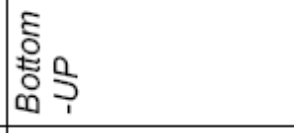 & 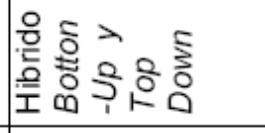 & 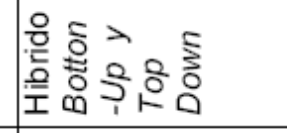 \\
\hline  & ¿্ঠ & ষ্ণ & 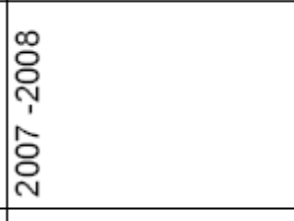 & ָָ & 을 \\
\hline 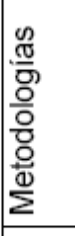 & 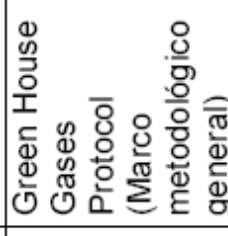 & 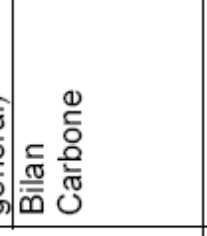 & 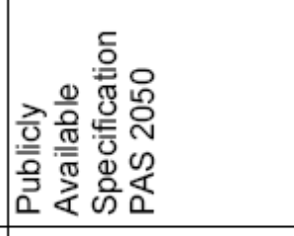 & 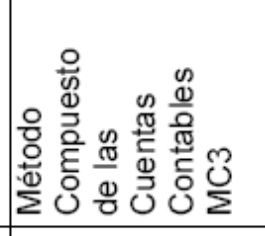 & 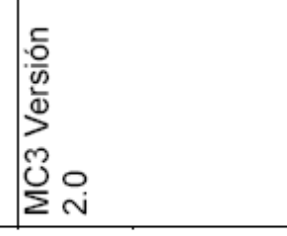 \\
\hline $\mid \frac{0}{\frac{0}{2}}$ & \multicolumn{2}{|c|}{ 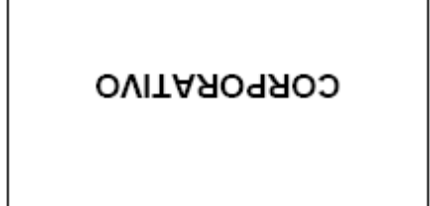 } & 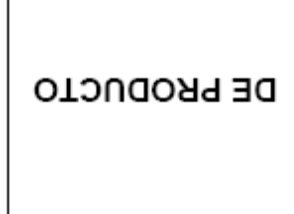 & \multicolumn{2}{|c|}{ 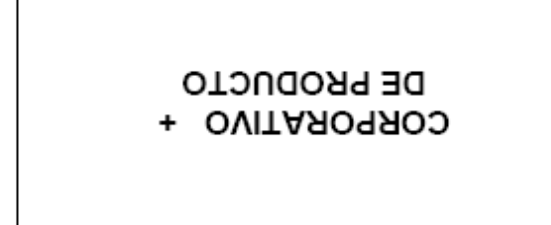 } \\
\hline
\end{tabular}


La Tabla 2 muestra una base comparativa para los características de los enfoques corporativo, de producto e híbrido mientras que la Tabla 3 muestra las características de las cuatro principales metodologías para determinar la HdC (GHG Protocl, Bilan Carbone, PAS 2050 y MC3. Esta información puede servir de base para la toma de decisiones gerenciales sobre la adopción de un determinado enfoque y metodología. Las licencias de uso de estas metodologías son gratuitas y de acceso abierto excepto la Bilan Carbone que entrega aplicaciones a personas que han seguido un proceso de capacitación con un costo por módulo (ADEME, 2010).

\section{La situación en Latino América}

En América Latina existe una serie de iniciativas en etapa inicial, a menudo en base a voluntades institucionales. Además, a pesar de la falta de uniformidad del marco metodológico disponible, empresas tanto locales como multinacionales con bases en América Latina, han implementado procesos de medición de Huella de Carbono hasta, en ciertos casos, lograr procesos de reducción y compensación para un estado "neutro en carbono". Estas experiencias demuestran los inicios de una concientización y reacción, que varía según los países y sus orientaciones económicas. El documento "Metodologías de cálculo de la Huella de Carbono y sus potenciales implicaciones para América Latina" de la CEPAL (2010) describe con cierto detalle la situación en Latino América con respecto a la HdC. La situación no ha cambiado en forma significativa en los últimos dos años. Un resumen de ese documento se describe en lo que sigue y la Tabla 4 sintetiza los principales organismos, legislación, estrategias, políticas, plan y programas estatales en algunos países latino americanos.

Brasil creó el año 2006, su propio mercado de carbono, el que tiene como meta principal el apoyo a la generación de proyectos MDL que podrán abastecer de bonos de carbono a los países desarrollados sujetos a cuotas de emisiones en el marco del Protocolo de Kyoto. Sin embargo, en el 2009 Brasil dio pasos concretos hacia compromisos propios de mitigación de emisiones de GEI. El Plan Nacional sobre el Cambio Climático (PNMC), que plasma los lineamientos de la lucha contra el cambio climático es ley federal desde el 9 de diciembre del 2009. La Ley adopta metas voluntarias de reducción de emisiones de GEI, entre 36 y 39 de las emisiones proyectadas para 2020 (Ro binson, 2010). Brasil está estructurando organismos y herramientas a fin de lograr esta meta, y ha entregado a los actores locales un fuerte mensaje de objetivos de mitigación. Brasil vive ahora una fase de crecimiento de la conciencia, sobre todo empresarial, y de iniciativas de medición y mitigación de la Huella de Carbono.

México tomó el compromiso de reducir al año 2012 sus emisiones de GEI en 50 millones de $\mathrm{tCO}_{2}$ equivalente, respecto a su nivel de emisiones de 2004, además de un compromiso general de reducción de $50 \%$ de las emisiones para 2050. Para cumplir esta meta diseñó e implementó el Programa Especial de Cambio Climático (PECC), su principal herramienta para identificar áreas vulnerables. El año 2008 el PECC integró el Programa Voluntario de GEl de México, implementado en el 2004, para la contabilidad de los GEI y para la generación de proyectos de reducción de emisiones. Al final del 2009 el programa contaba con 98 empresas inscritas, representando el $21 \%$ de las emisiones de GEl nacionales. En México, además de algunas iniciativas nacionales, tales como incentivo a la producción, y uso de biocombustibles en particular, se está dando un proceso interinstitucional de reforzamiento de la conciencia de los fuertes desafíos planteados por los impactos del calentamiento global.

Argentina ha mostrado fuertes preocupaciones con respecto a los avances y orientaciones demostrados por la Unión Europea y los Estados Unidos frente a los productos que estos países importan (etiquetado de carbono, potencial impuesto carbono). En particular, Argentina tiene motivaciones vinculadas con las exportaciones de productos agrícolas, argumentando que ciertas medidas podrían generar discriminación de sus productos frente a los mismos productos en otros ámbitos geográficos y otros contextos productivos. En el ámbito privado se desarrollan algunas iniciativas voluntarias en empresas multinacionales con actividades en Argentina (bancos, productos agrícolas) y empresas locales. En general, Argentina demuestra avances limitados en esta materia, especialmente a nivel institucional. 
Tabla 4: Principales organismos, legislación, estrategias, políticas, plan y programas estatales en algunos países latino americanos (adaptado de Cepal, 2010)

\begin{tabular}{|c|c|c|c|}
\hline País & Legislación & Estrategia/ /política & Plan/ /programa \\
\hline Argentina & $\begin{array}{l}\text { Ley } 25.675 \text { General de } \\
\text { Ambiente, del } 27 / 11 / 2002\end{array}$ & & $\begin{array}{l}\text { Programa nacional sobre } \\
\text { impactos del cambio climático }\end{array}$ \\
\hline Bolivia & $\begin{array}{l}\text { Ley del Medio Ambiente del } \\
27 \text { de abril de } 1992\end{array}$ & $\begin{array}{l}\text { Programa Nacional sobre } \\
\text { Impactos del Cambio } \\
\text { Climático }\end{array}$ & $\begin{array}{l}\text { Plan Nacional de Desarrollo } \\
\text { Programa Nacional de } \\
\text { Cambios Climáticos (PNCC) } \\
\text { Proyecto de Acción climática }\end{array}$ \\
\hline Brasil & $\begin{array}{l}\text { Ley } 6.938 / 1981 \text {, Dispone } \\
\text { sobre la Política Nacional de } \\
\text { Medio Ambiente }\end{array}$ & $\begin{array}{l}\text { Programa Nacional sobre } \\
\text { Impactos del Cambio } \\
\text { Climático }\end{array}$ & $\begin{array}{l}\text { Programa Nacional de Medio } \\
\text { Ambiente (I y II) Programa } \\
\text { Nacional de Mudanças } \\
\text { Climáticas (2008) }\end{array}$ \\
\hline Chile & $\begin{array}{l}\text { Proyecto de Ley para } \\
\text { modificar art. } 46 \text { de ley } \\
19496 \text { sobre protección de } \\
\text { los derechos del Consumidor }\end{array}$ & $\begin{array}{l}\text { Estrategia Nacional sobre } \\
\text { Cambio Climático }\end{array}$ & \\
\hline Colombia & $\begin{array}{l}\text { Ley } 99 \text { General Ambiental, } \\
\text { de } 1993\end{array}$ & $\begin{array}{l}\text { Lineamiento de Política } \\
\text { Nacional de Cambio } \\
\text { Climático del año } 2002\end{array}$ & $\begin{array}{l}\text { Planes Nacionales de } \\
\text { Desarrollo } 1998 \text { a } 2010 . \text { No } \\
\text { son específicos pero } \\
\text { mencionan el tema }\end{array}$ \\
\hline Costa Rica & $\begin{array}{l}\text { Ley Orgánica del Ambiente } \\
7554 \text { (1995) }\end{array}$ & $\begin{array}{l}\text { Estrategia Nacional de } \\
\text { Cambio Climático (ENCC) }\end{array}$ & $\begin{array}{l}\text { Plan Nacional de Desarrollo } \\
\text { (PND) } 2006 \text { - } 2010 \text { posiciona } \\
\text { la Agenda de Cambio Climático } \\
\text { como prioritaria }\end{array}$ \\
\hline Ecuador & $\begin{array}{l}\text { Ley No 37/1999 de Gestión } \\
\text { Ambiental }\end{array}$ & & \\
\hline El Salvador & $\begin{array}{l}\text { Ley } 233 \text { de Medio Ambiente } \\
\text { de } 1998\end{array}$ & & \\
\hline Guatemala & $\begin{array}{l}\text { DL 68-86, Ley de protección } \\
\text { y mejoramiento del medio } \\
\text { ambiente (1986) }\end{array}$ & $\begin{array}{l}\text { Política y Estrategia } \\
\text { Nacional de Cambio } \\
\text { Climático }\end{array}$ & $\begin{array}{l}\text { Programa Nacional del Cambio } \\
\text { Climático Plan Estratégico } \\
\text { Institucional del Ministerio de } \\
\text { Ambiente y Recursos Naturales } \\
\text { Administración } 2008-2012\end{array}$ \\
\hline México & $\begin{array}{l}\text { Ley General del equilibrio } \\
\text { ecológico y la protección } \\
\text { ambiental. (1998 y 2007) }\end{array}$ & $\begin{array}{l}\text { Estrategia Nacional de } \\
\text { Cambio Climático del año } \\
2007\end{array}$ & $\begin{array}{l}\text { Programa Especial de Cambio } \\
\text { Climático 2008-2012. } \\
\text { Programa Nacional de Medio } \\
\text { Ambiente y Recursos Naturales } \\
2007-2012 \text {. }\end{array}$ \\
\hline Nicaragua & $\begin{array}{l}\text { Ley } 217 \text { General de Medio } \\
\text { Ambiente y los Recursos } \\
\text { Naturales, de } 1996\end{array}$ & $\begin{array}{l}\text { Plan de Acción Ambiental, } \\
\text { marco de referencia de la } \\
\text { política y estrategia } \\
\text { ambiental oficial de } \\
\text { Nicaragua (1993) }\end{array}$ & $\begin{array}{l}\text { Plan de Acción Nacional ante } \\
\text { el Cambio Climático (PANCC), } \\
\text { elaborado en el } 2003\end{array}$ \\
\hline Paraguay & $\begin{array}{l}\text { Leyes } 1.561 / 2000,716 \text { de } \\
1996 \text { y } 294 / 93 \text { (Sistema } \\
\text { Nacional del Ambiente, } \\
\text { Sanciona delitos contra el } \\
\text { medio ambiente) }\end{array}$ & $\begin{array}{l}\text { Política Ambiental Nacional } \\
\text { (PAN) del año } 2005\end{array}$ & $\begin{array}{l}\text { Programa Nacional de Cambio } \\
\text { Climático dependiente de la } \\
\text { Secretaría del Ambiente, del } \\
\text { 2001. Plan quinquenal de } \\
\text { cambio climático 2008-2012 }\end{array}$ \\
\hline Perú & $\begin{array}{l}\text { Ley } 28.611 \text { General del } \\
\text { Ambiente, del } 13 \text { octubre de } \\
2005\end{array}$ & $\begin{array}{l}\text { Estrategia Nacional de } \\
\text { Cambios Climáticos. La } \\
\text { Agenda Ambiental Nacional } \\
\text { (2005-2007) Política } \\
\text { Nacional Ambiental (2009) }\end{array}$ & $\begin{array}{l}\text { Plan Nacional de Acción } \\
\text { Ambiental 2010-2021 (en } \\
\text { desarrollo) }\end{array}$ \\
\hline Uruguay & $\begin{array}{l}\text { Ley y No } 17 . .283 \text { General } \\
\text { de Medio Ambiente, , de } 22 \\
\text { de febrero de } 20000\end{array}$ & $\begin{array}{l}\text { Estrategia Ambiental } \\
\text { Nacional (no está } \\
\text { formalizada) }\end{array}$ & $\begin{array}{l}\text { Programa de Medidas } \\
\text { Generales para la Mitigación y } \\
\text { la Adaptación al Cambio } \\
\text { Climático. Sistema Nacional de } \\
\text { Respuesta al Cambio } \\
\text { Climático }\end{array}$ \\
\hline
\end{tabular}


Colombia está preocupada por los impactos y costos de adaptación que pueden significar las orientaciones europeas y estadounidenses en materia de emisiones y HdC. Sin embargo, tanto a nivel estatal como de la sociedad se observa que la concientización ha ido avanzando habiendo iniciativas relacionadas al tema, como es el caso del estudio sobre la HdC de flores de exportación a Europa. Los avances más significativos en términos de $\mathrm{HdC}$ se observan a través de iniciativas voluntarias privadas y de la sociedad civil. La posición del gobierno ha sido de espera y negociación de los impactos y soporte de gastos potencialmente generados por la implementación de las medidas proyectadas en Europa y los Estados Unidos. A nivel privado se han desarrollado distintas iniciativas voluntarias como la medición de la HdC en empresas y comunidades e iniciativas de compensación en empresas petroleras.

Chile ha empezado un proceso institucional de análisis de adaptación/respuesta a los desafíos planteados por las orientaciones europeas y estadounidenses, con la visión clara de lograr mantener y fortalecer su competitividad en el escenario internacional frente a vecinos latinoamericanos o países de otras regiones. En particular, en mayo de 2009 inició el estudio "Huella de Carbono en productos de exportación agropecuarios de Chile", que se desarrolla en el marco del Plan de Acción Nacional del Cambio Climático 2008-2010, que ha incluido de forma explícita la $\mathrm{HdC}$ en las consideraciones de orden estratégico para enfrentar el cambio climático.

En paralelo, empresas grandes y medianas han empezado procesos voluntarios de cálculo de sus emisiones de GEl a nivel de sitios de producción. Este es el caso del sector forestal, maderero y papelero, donde varias empresas implementan medidas de reducción y compensación. En el sector minero se está desarrollando un proyecto en el cual se busca reconocer potenciales emisores de GEl que pueden ser reducidas en sus faenas. El sector más avanzado en Chile es el vitivinícola, situación que se explica, entre otros motivos, por la presión que ejercen algunos importadores europeos, su principal mercado. Las herramientas metodológicas utilizadas son, en la mayoría de los casos, replicas o adaptaciones del GHG Protocol y del PAS 2050.

Aunque en Chile todavía no se dispone de todas las herramientas necesarias, el país ha establecido las bases de procesos de avance en su reflexión hacía la adaptación de estrategias y herramientas dedicadas a la Huella de Carbono, con la motivación de mantener los niveles de exportaciones hacia Europa y los Estados Unidos, y la expectativa de presiones frente a su entrada en la Organización para la Cooperación y el Desarrollo Económico (OCDE). Chile se posiciona como uno de los líderes en América Latina con respecto a la Huella de Carbono.

La mayoría de los países de América Latina considera a la HdC y las decisiones que pueden resultar de ella, como un impuesto al carbono y como potencialmente discriminatorias en el marco de los intercambios comerciales de productos agrícolas, materias primas et al.. Consideran, por otra parte, que los países y regiones reconocidos como responsables del calentamiento global (países industrializados), deberían apoyarlos en mejorar sus tecnologías y conocimientos en esta materia para que no sean castigados en forma "unilateral". Los procesos voluntarios como la HdC de productos se sigue interpretando a menudo como un factor comercial y de marketing, propio de las empresas que pueden estar sometidas a una presión eventual, y no como una política de Estado en respuesta a desafíos económicos internacionales. Considerando que ciertos países están más avanzados que otros, las debilidades de los países latinoamericanos frente a la implementación de la HdC y sus consecuencias son, según el documento de la CEPAL (2010), las siguientes:

- Falta de conocimiento técnico en las fuentes de emisiones de GEl y su medición;

- Falta de análisis crítico de los marcos metodológicos en los países compradores;

- Falta de análisis de necesidad de elaboración de marcos metodológicos propios

- Falta de conocimiento y desarrollo de los factores de emisión que se podrían aplicar a la región

- Falta de identificación de los actores, procesos y certificados que validen la medición de la HdC;

- Falta de armonización de posiciones frente a medidas tales como el Impuesto Carbono

- Falta de difusión/educación hacia la sociedad civil, las empresas y el público/cliente en general;

- Falta de preparación y anticipación de futuras regulaciones en los sectores públicos y privados. 
Como se ha descrito anteriormente, a pesar de que los países desarrollados están más avanzados al respecto, aún no han solucionado integralmente algunas de las debilidades a las que son sujetos. Para los países de Latinoamericanos se abre un importante espacio de discusión y negociación, especialmente para aquellos de economías abiertas bajo un modelo económico eminentemente exportador. En este sentido, parece relevante abordar desde Latinoamérica procesos de investigación destinados a resolver interrogantes frente a comparación de enfoques metodológicos de cálculo, con el objeto de clarificar las vulnerabilidades de las exportaciones por futuras exigencias sobre la $\mathrm{HdC}$.

\section{PROPUESTAS DE TRABAJO}

Un profesional que deba tomar una decisión sobre como cuantificar las emisiones de la empresa con fines de etiquetado, verificación o certificación de emisiones, bajo un proceso voluntario o inducido por necesidades competitivas, enfrenta hoy en día un escenario de incertidumbres sobre el que es prácticamente imposible trabajar. La sola tarea de informarse, evaluar y decidir sobre que enfoque metodológico a adoptar, requeriría de un estudio acabado que involucra una considerable cantidad de tiempo y dinero. Sin embargo, si se dispone de una base de información resumida en base a tres importantes subconjuntos de información fundamental se podría facilitar dicho proceso de decisión. Estos tres subconjuntos de información son: i) las bases conceptuales; ii) la naturaleza de la información requerida en el proceso; y iii) el ámbito para el que está dirigido el cálculo de la HdC. Considerando los diferentes enfoques y creyendo en la necesidad de ir integrando estas visiones para disponer a futuro de un procedimiento universalmente aceptado se propone dos herramientas complementarias que permiten ir avanzando en la comprensión y aplicación de las metodologías disponibles: i) el ábaco para la contabilización de emisiones y ii) la relación entre las metodologías disponibles.

\section{Ábaco para la contabilización de emisiones de $\mathrm{CO}_{2}$ equivalente}

Como contribución a la comprensión de los diferentes métodos, y con el objetivo de visualizar en mejor forma las similitudes y las diferencias entre ellos, permitiendo un mejor análisis de una situación donde se necesite aplicar el concepto de $\mathrm{HdC}$, se propone en este trabajo el Ábaco para la contabilización de emisiones de CO2-eq. Este ábaco que se muestra en la Fig. 1 incluye una serie de conceptos que es necesario definir. En forma general, el concepto HdC, puede ser presentado de una manera simple considerando tres aspectos fundamentales: i) definir el enfoque del análisis (identificación de cómo se enfocará el análisis y el alcance considerado para determinar la $\mathrm{HdC}$ ); ii) clasificar las emisiones que serán consideradas en el cálculo de la HdC y que dependen del método a usar para su cálculo; y iii) definir el formulismo para el cálculo de la $\mathrm{HdC}$ que conduce a la magnitud del indicador. Esta descripción que se muestra gráficamente en la Fig. 1, facilitará la comprensión del concepto y su aplicación por parte de los gestores a una situación o unidad de análisis concreta.

El enfoque del análisis empleado, se refiere a definir el sistema foco del análisis. Por ejemplo si se considera la empresa completa, o un proceso en particular, o solamente los procesos productivos, o los productos principales. En esto hay varios enfoques que usualmente se engloban en dos grandes conceptos: enfoque de arriba abajo (top down) o enfoque corporativo y enfoque de abajo a arriba (bottom up) o enfoque de producto. El enfoque de arriba abajo o enfoque corporativo parte desde una visión global de una organización, para la obtención de una o más magnitudes específicas de HdC. El enfoque de abajo a arriba o enfoque de producto en el que se realiza un análisis muy específico de la magnitud de unidades constituyentes de una organización, evento o proceso e ir agregando magnitudes parciales específicas, hasta la obtención de una magnitud global de $\mathrm{HdC}$ para la unidad en estudio, generalmente un producto o un servicio.

La identificación y clasificación de las emisiones puede ser explicada considerando tres elementos comunes a cualquiera de los métodos en estudio. Por una parte, todo cálculo de la HdC requiere en la práctica de la definición de subconjuntos para la clasificación de emisiones, que se puede llamar Organizador de Emisiones. 


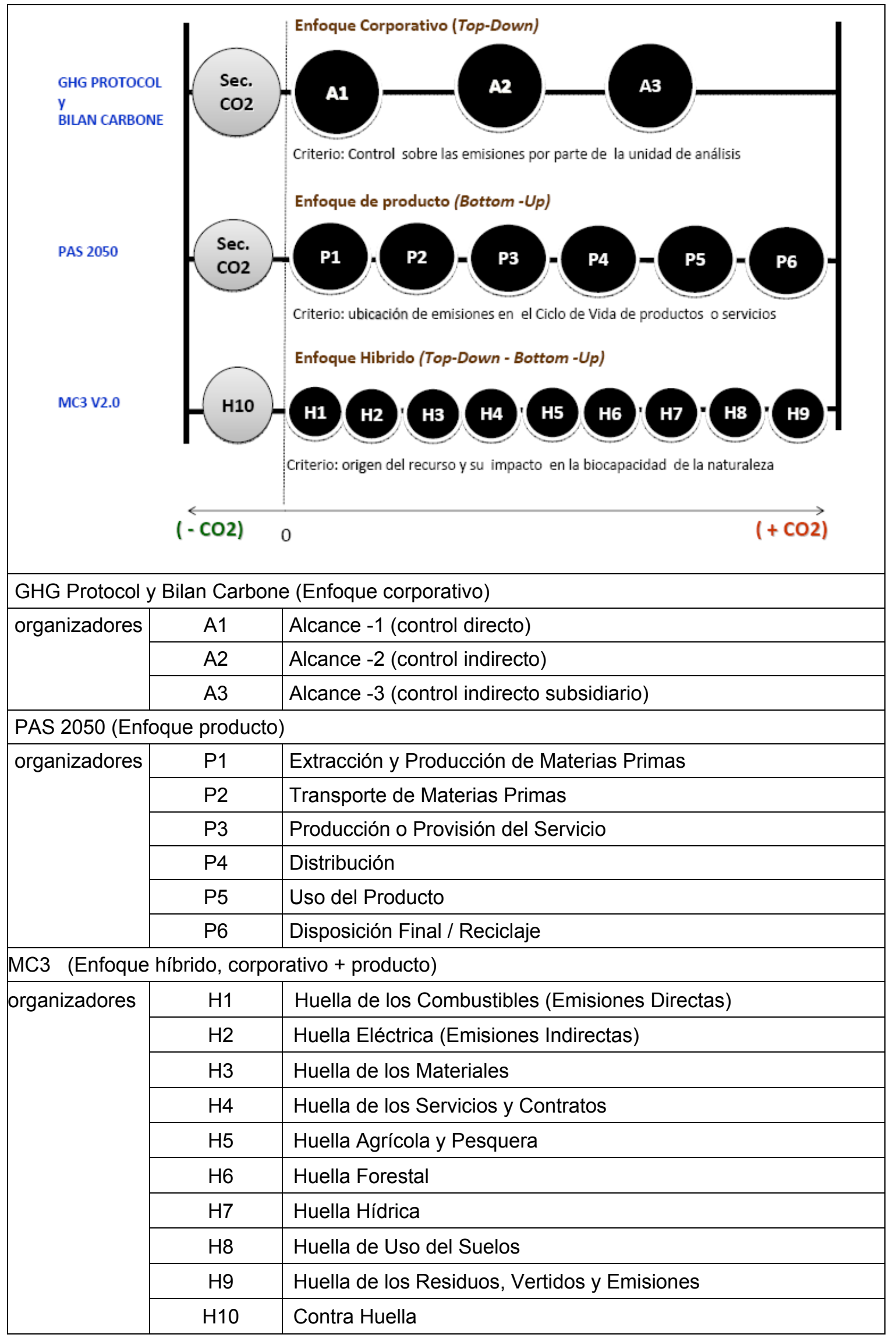

Fig. 1: Ábaco para la contabilización de emisiones $\mathrm{CO} 2$ equivalentes 
Un segundo aspecto a considerar corresponderá a aquellas cualidades o atributos que determinan la pertenecía o no al subconjunto de emisiones y que se denomina Criterio de Clasificación de Emisiones. Si se observa en Abaco propuesto los enfoques corporativos GHG Protocol y Bilan Carbone, el Organizador $A_{i}$ o conjunto de emisiones recibe el nombre de Alcance y su proceso de clasificación está basado en el Criterio de Control. Esto es que la pertenecía a cada uno de los alcances depende del grado de control de la unidad de análisis sobre las emisiones de $\mathrm{CO}_{2}$-eq. Este control puede ser Directo (A1), Indirecto (A2) o Indirecto Subsidiario (A3). El organizador A1 controla las emisiones directas provenientes del uso de combustible bajo control de la empresa; A2 controla las emisiones indirectas sólo por consumo de electricidad adquirida; y A3 controla las emisiones subsidiarias o indirectas fuera del control de la empresa).

En el caso de los métodos PAS 2050 y del futuro ISO14067 el conjunto organizador se denomina Proceso $\left(P_{i}\right)$ y su criterio de selección corresponde a la Etapa del Ciclo de Vida de Producto en la cual se generan las emisiones. Este método en particular considera 6 conjuntos organizadores correspondientes a seis procesos genéricos descritos en la Fig. 1. Finalmente en el caso de la metodología MC3 se consideran nueve conjuntos organizadores de las emisiones denominados Huellas $\left(\mathrm{H}_{\mathrm{i}}\right)$ y cuyo criterio de clasificación se denomina Categoría de Recursos Consumibles asociados directamente al origen del recurso natural que está siendo utilizado por la unidad de análisis. En este caso en particular la conversión está dada a partir de unidades monetarias en $\mathrm{CO}_{2}$-eq, mientras que en los otros métodos corresponden a transformaciones desde unidades de masa, volumen y energía en unidades de $\mathrm{CO}_{2}$-eq .Tanto el número de organizadores como los criterios de clasificación pueden diferir entre uno y otro método dependiendo del enfoque tal como lo muestra la Fig. 1.

El tercer elemento que aparece de manera implícita en los métodos de cálculo se denomina Sentido de la Magnitud y determina el incremento o diminución de la magnitud de la HdC, ya sea producto de la identificación de emisiones antropogénicas (aumentos de magnitud +CO2) o determinación de capacidades de absorción o secuestro de emisiones (disminuciones de magnitud $-\mathrm{CO}_{2}$ ). Este último caso asociado fundamentalmente a cambio de uso de suelos no agrícolas en agrícolas o bien actividades como tratamientos silviculturales para aumentar el crecimiento, agroforestación, forestación, reforestación y restauración de áreas degradadas que el productor tiene a su haber como en el espacio destinado a la producción. En el Abaco de la contabilización de emisiones esto se traduce en la incorporación de un subconjunto adicional que no es explícito en todos los métodos, en especial en aquellos basados en enfoques corporativos o de productos y que ha sido expresado en el ábaco como secuestro de $\mathrm{CO} 2\left(\mathrm{Sec} . \mathrm{CO}_{2}\right)$. En el caso del enfoque Hibrido planteado por la metodología MC3 no sólo se especifica como Contra huella (H10) sino que además es propuesto como un importante indicador a considerar en una gestión sustentable, incentivando la inversión en capital natural por parte de las organizaciones (Dómenech et al., 2010).

Desde la perspectiva latinoamericana, la adopción voluntaria de metodologías basadas en enfoques híbridos como MC3, podría acelerar los procesos de implementación de prácticas de gestión amigables con el medio ambiente, como la HdC. Esto porque es menos compleja en términos del manejo de información ya que se basa en información contable (unidades monetarias), disponible en cualquier organización y que es condición necesaria para su funcionamiento formal como unidad productiva o de servicios. Esta ventaja metodológica podría reducir significativamente las barreras para su adopción como práctica de gestión, al requerir menores esfuerzos técnicos y económicos para la cuantificación de la HdC. Sin embargo, cuando el mercado de destino de la producción, está en el mercado internacional, la elección del enfoque metodológico asociado al cálculo de la $\mathrm{HdC}$ estará necesariamente supeditada a la exigencia de su contraparte comercial.

\section{Relación entre las metodologías disponibles}

Si bien el Ábaco para la contabilización de emisiones puede aportar al proceso de adopción de la $\mathrm{HdC}$ como una práctica de gestión empresarial, aún seguirá pendiente el desarrollo de esfuerzos por establecer avances en la comparabilidad de magnitudes de $\mathrm{HdC}$ obtenidas por diferentes 
marcos metodológicos. Esto cobra gran importancia en el ámbito de la competitividad internacional, en especial, cuando por desconocimiento o intencionadamente estos métodos son utilizados indiscriminadamente y cuyas magnitudes son incorporadas en estrategias de comunicación corporativa o como estrategia de diferenciación frente a productos de la competencia, situación que complica los escenarios de competitividad en el ámbito internacional. Este problema que se hace especialmente complejo en el caso de países como los latinoamericanos que reciben estos marcos metodológicos como estándar competitivo, participando marginalmente de los procesos de discusión internacional. Considerando lo anterior, parece evidente que la $\mathrm{HdC}$ corporativa debiese estar relacionada en forma directa con la $\mathrm{HdC}$ de los productos de una misma corporación y que considerando una misma unidad de análisis se pudiese encontrar un factor de equivalencia $\alpha$ entre ambos enfoques de cálculo, por lo tanto se propone la relación mostrada en la ecn. (1):

Hdc corporativo $\cong \alpha \sum \mathrm{HdC}$ de producto $\mathrm{i}$ para $\mathrm{i}=1 \ldots \mathrm{n}$

Para una organización que ha optado por un método específico como PAS 2050, para etiquetar sus productos con una magnitud de $\mathrm{HdC}$ y que se enfrenta a un producto competidor que exhibe en su etiqueta una magnitud, en teoría equivalente obtenida por un método corporativo como Bilan Carbone, sería muy conveniente conocer que tan objetivo es el cálculo exhibido por su competidor. De igual forma desde el punto de vista de un consumidor, este esperaría tener antecedentes de cuál de las dos magnitudes que aparentemente son iguales, subestima los impactos ambientales que declara, dado que dicha magnitud de $\mathrm{HdC}$ en definitiva se traduce en un atributo para la asignación de valor al producto y que influye en proceso de decisión de su compra. Es así como deberíamos aspirar a obtener factores $\beta \mathrm{k}$ que identifiquen las equivalencias entre las diversas combinaciones de pares métodos disponibles tal como como se describe en la ecn. (2):

$\mathrm{HdC}$ producto Bilan carbone ${ }^{\circledR}=\beta \mathrm{k} \cdot \mathrm{HdC}$ producto PAS 2050

En otras palabras, debiésemos aspirar a tener una misma magnitud de impactos ambientales, pero expresada en diversas escalas, con factores de equivalencia conocidos, tal como expresamos hoy la magnitud de la temperatura en escalas de grados Celcius a Fahrenheit, ejemplo que parece ad hoc, cuando el indicador de $\mathrm{HdC}$ no hace más que predecir indirectamente el potencial cambio de la temperatura global. Estas aspiraciones se ven reforzadas con cada oportunidad que se desperdicia para establecer acuerdos globales frente emisiones de $\mathrm{CO}_{2}$ y con la proliferación de iniciativas y metodologías de cálculo de orden nacional. Esta situación nos permite predecir que con independencia de la aprobación de un estándar internacional como la ISO 14067 estaremos obligados a convivir con diversos marcos metodológicos para el cálculo de la $\mathrm{HdC}$, que poco a poco comienzan a instalarse como marcos regulatorios nacionales.

La identificación de los parámetros a y $\beta$ k podrá ser determinada y generalizada cuando se disponga de mayor información producto de estudios empíricos de diversos marcos metodológicos sobre una misma unidad de análisis. En términos de la contribución a la gestión empresarial la determinación de los valores a y $\beta$ k podría aportar a la identificación de una mayor o menor incertidumbre y objetividad respecto de los impactos ambientales involucrados en un determinado método de cálculo, así también podrían constituirse en indicadores de los niveles de exposición o vulnerabilidad competitiva causada por la mala selección de un método de cálculo de HdC, Esto podría permitir a la HdC consolidar su posición en la estrategia competitiva de las empresas.

\section{CONCLUSIONES}

Del estudio realizado, del análisis de la información de la literatura, y de la visión propuesta para la toma de decisiones se pueden obtener las siguientes principales conclusiones: 
1.- El éxito en la contribución al cambio climático que pueda ofrecer la huella de carbono para poner en práctica cadenas de producción sostenibles, pasa por el reconocimiento de las responsabilidades de productores y de consumidores

2.- De mantenerse esta falta de claridad y comparabilidad en la determinación de emisiones podría provocar a la larga el fracaso de un eco-etiquetado global y la pérdida de una gran oportunidad para lanzar una nueva economía sostenible

3.- Los exportadores se enfrentan a un doble problema, ya que al enviar productos a más de un mercado deben asumir el desafío de responder simultáneamente a requerimientos muy diversos en términos de $\mathrm{HdC}$

4.- En el ámbito del comercio internacional hay dos enfoques que han cobrado mayor importancia: el enfoque de producto basado fundamentalmente en el análisis el ciclo de vida de dicho producto (PAS 2050) y el enfoque corporativo (GHG Protocol y Bilan Carbone). Aunque parece atractivo el método MC3 que no ha tenido amplio uso, a pesar de sus ventajas, como se explicó en la Parte 1 de esta serie.

5.- Ambos enfoques metodológicos, corporativo y producto) podrían generar importante repercusión a corto plazo en los países de América Latina, fundamentalmente por el carácter eminentemente exportador de recursos naturales y productos de bajo valor agregado.

\section{AGRADECIMIENTOS}

Los autores agradecen el apoyo de la Universidad de La Serena y del Centro de Información Tecnológica (Chile) para la realización de este trabajo. CE agradece también al Depto. Adm. Empresas y Gestión Económica de Rec. Naturales, de la Universidad de Lleida (España).

\section{REFERENCIAS}

Ademe (l'Agence de l'Environnement et de la Maîtrise de l'Energie). Bilan Carbone®, Entreprises et Collectivités. Guide méthodologique, version 6.1, objectifs et principes de comptabilisation (2010).

Boiral, O. Corporate Response to Global Warming: For a Proactive Strategy. International Journal of Business and Economics Perspectives, 1(1), 79-95 (2006).

Bolwig, S. y P. Gibbon. Emerging product carbon footprint standards and schemes and their

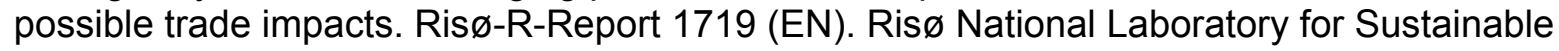
Energy, Technical University of Denmark (DTU), ISBN:978-87-550r-r3796-0 (2009).

Brenton, P. G. Edwards-Jones y M.F. Jensen. Carbon labelling and low income country exports: A review of the development issues. Development Policy Review, 27, 243-265 (2009).

BSI, Bristish Standards Institute. PAS 2050: 2008. Specification for the assessment of the life cycle greenhouse emissions of goods and services, 2008. Disponible en http://www.bsigroup.com/ en/Standards-and-Publications/Industry-Sectors/Energy/PAS-2050/. Acceso en 20.12 (2010).

Bristish Standards Institute (BSI). PAS 2060: Specification for the demonstration of carbon neutrality, 2010.Disponible en http://shop.bsigroup.com/en/ProductDetail/?pid= 000000000030198309 . Acceso en 20.03 (2011).

CEPAL, Comisión Económica Para América Latina y El Caribe. Metodologías de cálculo de la Huella de Carbono y sus potenciales implicaciones para América Latina (2010).

De La Torre, A. P. Fajnzylber y J. Nash. Desarrollo con menos carbono: respuestas latinoamericanas al desafío del cambio climático. Banco Central, Washington D.C., USA (2009). 
Doménech, J.L., A. Carballo, L. Jiménez y J.L. De La Cruz. Estándares 2010 de Huella de Carbono MC3. In: Conama10 Congreso Nacional de Medio Ambiente, 11,2010. Anais. Madrid, España (2010).

Ernest \& Young, Cambio Climático - Huella de carbón 2010. Disponible en http://www.prochile.cl/ regiones_pro/archivos/region_VIII/PRESENTACION\%20Ernst\%20\&\%20Young.pdf Acceso en $15.12(2010)$.

Foran, B. M., M. Lenzen, D. Christofer y M. Bilek. Integrating sustainable chain management with triple botton line accounting. Ecological Economics, 52,143-157 (2004).

Jancovici J. M. Un outil pour connaître les émissions de gaz à effet de serre d'une entreprise ou administration : le "bilan carbone" de l'ADEME,2003. Disponible en http://www.manicore.com/missions/bilan_carbone.html. Acceso en 01.03 (2011).

Kasterine, A. y D. Vanzetti. The effectiveness, efficiency and equity of market-based and voluntary measures to mitigate greenhouse gas emissions from the agri-food sector, United Nations Conference on Trade and Development. Trade and Environment Review 2009/2010 New York/Geneva (2010).

Macgregor, J. y B. Vorley. Fair Miles? The concept of "food miles" through a sustainable development lens. Sustainable Development Opinion. IIED, London, UK (2006).

Plasmann, K. A. Norton, N. Attarzadech, M.P.Jensen, P. Brenton y G. Edwards-Jones. Methodological complexities of product carbon footprinting: a sensitivity analysis of key variables in a developing country context. Environmental Science \& Police, 13, 393-404 (2010).

Robinson, K. Brazil's Global Warming Agenda. Obtenido de wri.org. http://www.wri.org/stories/ 2010/03/brazils-global-warming-agenda (2010).

Roos, E., C. Sundberg y P. Hansson. Uncertainties in the carbon footprint of food products: a case study on table potatoes. The International Journal of Life Cycle Assessment 15, 478-488 (2010).

Schneider, H. y J.L. Samaniego. La huella del carbono en la producción, distribución y consumo de bienes y servicios, documentos de proyectos, $N^{\circ} 298$, Santiago de Chile, Comisión Económica para América Latina y el Caribe (2009).

SETAC, The Society of Environmental Toxicology and Chemistry (SETAC), Standardisation Efforts to Measure Greenhouse Gases and Carbon Footprinting for Products, Int. J. of Life Cycle Assessment, 13(2) 87-88 (2008).

Sinden, G. The contribution of PAS 2050 to the evolution of international greenhouse gas emission standards. Int. J. of Life Cycle Assessment, 14, 195-203 (2009).

Wiedmann, T. Editorial: Carbon Footprint and Input-Output Analysis. An introduction, Economic Systems Research, 21(3), 175-186 (2009).

Wiedmann, T.; J. Minx, A Definition of Carbon Footprint In: C. C. Pertsova, Ecological Economics Research Trends: Chapter 1, p. 1-11, Nova Science Publishers, Hauppauge NY, USA. (2008).

Wiedmann, T; Barrett, J.; Lenzen, M. Companies on the Scale: Comparing and Benchmarking the Footprints of Businesses. International Ecological Footprint Conference. Cardiff, 8-10 May (2007).

Wittneben, B.B.F; Kiyar, D. Climate change basics for managers, Management Decision, 47(7), 1122-1132 (2009). 
